\title{
Design of Micro Robot for Minimally Invasive Surgery
}

\author{
Deiva Ganesh A \\ Departement of Mechanical Engineering, Velammal Engineering College, Chennai, India
}

\begin{tabular}{|c|c|}
\hline Article Info & ABSTRACT \\
\hline Article history: & \multirow{10}{*}{$\begin{array}{l}\text { Micro robots for medical applications need to be compatible with human } \\
\text { body, remotely controllable, smooth in movement, less painful to the patients } \\
\text { and capable of performing the designated functions. In this paper, state of the } \\
\text { art in the design, fabrication and control of micro robots are presented. First } \\
\text { the benefits of micro robots in medical applications are listed out. Second, } \\
\text { the predominantly used micro robot designs are discussed. Third, the various } \\
\text { fabrication process used in micro robot construction are presented. Fourth, } \\
\text { the different approaches used for its operation and control in micro robot } \\
\text { technology are narrated. Next based on the review we have designed a } \\
\text { swimming micro robot driven by external magnetic fields for minimally } \\
\text { invasive surgery. The advantage of EMA is that it can generate a wireless } \\
\text { driving force. Then, the locomotive mechanism of the micro robot using } \\
\text { EMA is presented. Using the EMA system setup various experiments have } \\
\text { been conducted. Finally, the performance of the swimming micro robot is } \\
\text { evaluated. }\end{array}$} \\
\hline Received Aug 13, 2012 & \\
\hline Revised Nov 1, 2012 & \\
\hline Accepted Nov 27, 2012 & \\
\hline \multirow{7}{*}{$\begin{array}{l}\text { Keyword: } \\
\text { Biomedical Robotics } \\
\text { EMA Robot } \\
\text { MEMS } \\
\text { Micro robot } \\
\text { MIS }\end{array}$} & \\
\hline & \\
\hline & \\
\hline & \\
\hline & \\
\hline & \\
\hline & $\begin{array}{r}\text { Copyright }(2013 \text { Institute of Advanced Engineering and Science. } \\
\text { All rights reserved. }\end{array}$ \\
\hline \multicolumn{2}{|l|}{ Corresponding Author: } \\
\hline \multicolumn{2}{|l|}{ Deiva Ganesh A, } \\
\hline \multicolumn{2}{|c|}{ Departement of Mechanical Engineering, } \\
\hline \multicolumn{2}{|c|}{ Velammal Engineering College, Chennai - 600066, } \\
\hline \multicolumn{2}{|c|}{ Tamil Nadu, India, Email- Id : amdganesh@yahoo.co.in } \\
\hline
\end{tabular}

\section{INTRODUCTION}

Lot of research work on micro robots for medical application has been continuing towards the ongoing efforts to decrease damage to human body during an operation and to reduce operation time. Since the 1980's, medicine has seen a dramatic shift towards the use of minimally invasive procedures because of the many advantages this technology presents $[10,12]$. Amongst various applications, micro robots that can move along blood vessels and treat specific parts of body have received much attention. The ultimate objective of the research is to design a robot that can reach the destination accurately and quickly [29].

Minimally invasive procedures are linked with a variety of patient-oriented benefits ranging from reduction of recovery time, medical complications, infection risks, and post-operative pain to increased quality of care, including preventative care $[4,8]$.

Bradley and Nelson[1] describe that the operations performed by micro robots will potentially entail several different steps: a) processing of previously acquired medical data (primarily images), simulation and planning of interventions; b) design of the optimal configuration of the micro robot customized for the specific patient anatomy and for the planned therapy at the target site; c) delivery of devices within the body to the desired site; d) extremely precise execution of the intervention; e)disassembly, recovery or biodegradation of the devices [42-47]. In this paper, the literatures related to design, fabrication and actuation have been discussed and a new micro robot design is presented.

Generally, small motors and smart materials are used as actuators for the micro robot. However, they increase the size of the micro robot prohibiting it from being used for minimally invasive surgical applications. To solve this problem, electromagnetic based actuation (EMA) systems for micro robot were used [30-40].

The paper is organized as follows. Section one gives an introduction to the use of micro robots for minimally invasive surgery. In section two, various micro robot designs are discussed. In the third section, 
micro robot fabrication techniques are presented. Section four gives the control of the micro robot. In the fifth section a new design of a swimming micro robot using EMA coil system is presented. The sixth section gives the analysis of the movement. The final section details the experiments and results.

\subsection{Micro Robot Designs}

Micro-robots for medical use can be categorized into two main groups, those that are designed for swimming and those that crawl, gripping the inner pipe walls. The first group might suit medical applications where almost no flow is applied on the robot, while crawling micro-robots can be applicable to flow where massive bloodstream flow present in the human blood vessels [2, 14].

Bradley and Nelson [1, 16] spelt out on the challenging design issues present themselves when envisioning a medical micro robot for in-vivo applications. Devices must be small, reliable and biocompatible, must carry the necessary tools and subsystems on-board and must be inserted into, steered inside and removed from the target area of the patient's body in a "minimally-invasive" way. It is difficult to resolve all these issues at once, also because much depends on the particular application.

A schematic diagram of the medical micro robot is shown in Figure 1. Zhoul and Quanl [2], [26] designed a medical micro robot consists of a right spirally grooved micro motor, a left spirally grooved cylinder and a flexible coupling as shown in Figure 1.

The new robot shown in Figure 2 consists of a central torso from which tiny arms stretch out, allowing the robot to strongly grip the vessel walls $[5,9]$. The operator can manipulate the robot to move in increments, and its unique structure allows it to crawl within a variety of vessels with differing diameters.

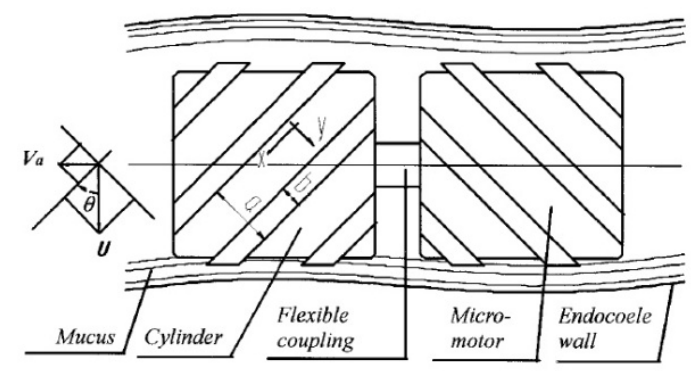

Figure 1. Schematic diagram of medical micro robot by Zhoul and Quanl $[2,26]$

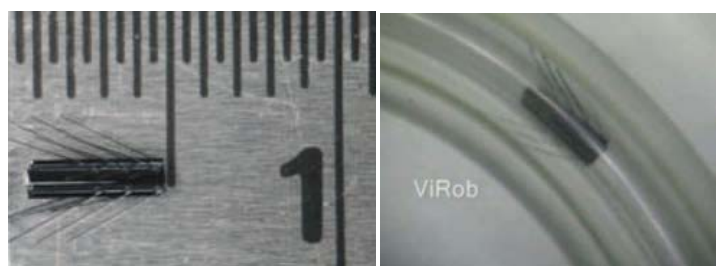

Figure 2. An autonomous crawling Micro- robot

One of the applications of a flagellar swimmer is interventions in the ventricular system in the brain. Kosa and Jakab [3] presented a swimming micro robot. Figure 3 illustrates the introduction of the swimming robot into the ventricular space.

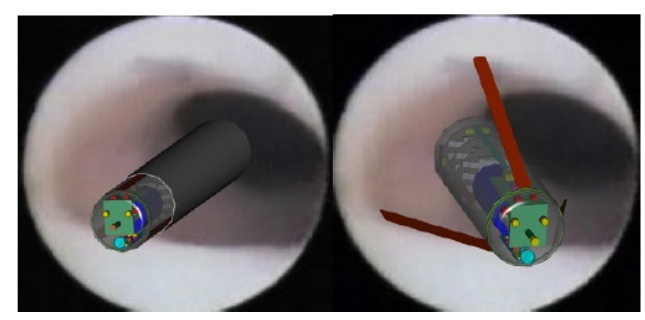

Figure 3. Illustration of the Swimming micro robot by Kosa and Jakab [3] 
The critical components in the robot are the following: three flagellar swimming tails, power source and custom designed IC

\subsection{Micro Robot fabrication}

One enabling technology for medical micro robots is Micro-Electro-Mechanical-Systems (MEMS). Usually, it is a combination of their low cost, low power consumption and small size that makes a MEMS based design the better choice compared to conventional technology [13].

Behkam and Sitti [7] presented the micro fabrication processes, such as micromachining, that typically use lithography, although other non-lithographic precision micro fabrication techniques exist (FIB, EDM, laser machining). Kovacs [24] and Madou [25, 27] provide a comprehensive discussion of micro machining processes and MEMS devices.

Another design challenge for a sub-mm sized micro robot is the high degree of integration [1-6]. Yesin and Nelson [26] have presented the emerging technology of Hybrid MEMS, where individual MEMS components are combined through a robotic micro assembly process, promises a solution.

\subsection{Control of Micro Robot}

The important and challenging part of this technology is control of the micro robots. Magnetic and Piezo-electric actuations are widely used for realizing micro robot motion and are discussed below [41].

Magnetic actuation technology has been applied in biological systems for many years when wireless actuation is needed.

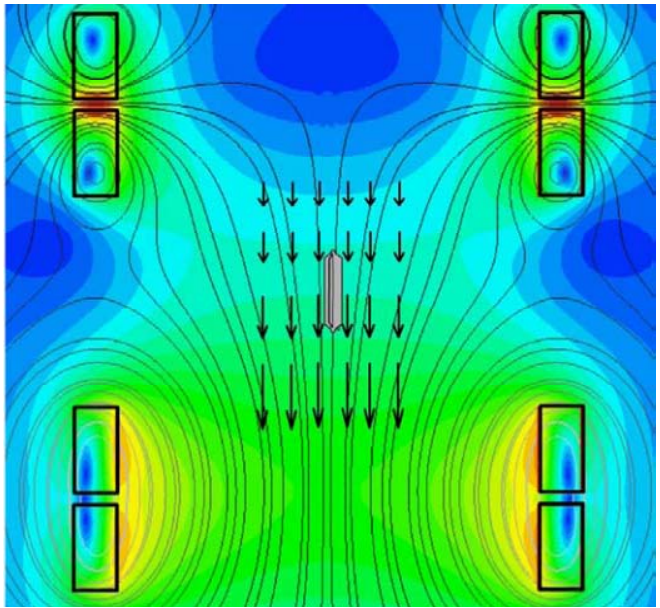

Figure 4. Superimposed magnetic field generated by Maxwell and Helmholtz coils.

A similar configuration called the Maxwell coil can generate a uniform gradient near the centre. Figure 4 shows superimposed magnetic field generated by concentric Helmholtz and Maxwell coils. This configuration enables independent control of magnetic force (thrust) and torque (orientation) on the micro robot [50-58]. Both of these coil types are commonly used in MRI systems. Recent efforts are towards applying this principle in a larger scale in combination with on-board magnetic actuators [48, 49].

\section{RESEARCH METHOD}

Generally, because a micro robot has an actuator, and control electronic circuits, the volume of the micro robot is increased. A large micro robot is difficult to apply to a human body in medical applications. However, the micro robot using the EMA system can be miniaturized by a small size permanent magnet, which is located in the micro robot's body.

Guo suggested a simple swimming micro robot [14-20] which has a magnetic fin attached the magnet directly. The micro robot moves along a pipe, which is surrounded by a coil. However, it can move only inside the coiled pipe and cannot be applied to the human body.

Masahiro suggested a turning fish type micro robot [21-25] using a magnet. An external magnetic field is generated by the coil. A magnet in the fish type robot is wire connected with the fin. But this type cannot be made small due to the wire connection and it is difficult to control precisely [28]. 
In this paper a new design of swimming micro robot is proposed. It has a simple structure and it can be controlled by EMA system with two pairs of Helmholtz coils. The swimming motion of the micro robot can be changed by controlling the swing angle of the fin and the frequency of the swing motion.

\subsection{EMA Coil System}

Generally, a pair of Helmholtz coils is used to generate a uniform magnetic field in the region of interest (ROI). When a permanent magnet is located in the uniform magnetic field generated by a Helmholtz coil, it rotates to align in the direction of the generated uniform magnetic field and the following torque is generated [43].

$$
\tau=\mathrm{VM} \times \mathrm{B}
$$

Where $\mathrm{V}$ and $\mathrm{M}$ are the volume and the magnetization of the permanent magnet and $\mathrm{B}$ denotes the magnetic flux of the external magnetic field.

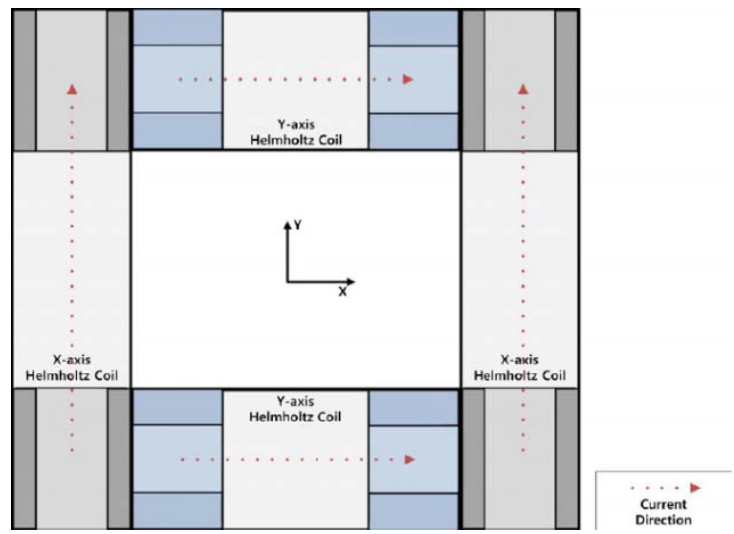

Figure 5. EMA System

\subsection{New Design of Micro Robot}

As shown in Figure 6 a simple swimming micro robot with a simple propulsion mechanism in the form of a swing fin attached at the rotating magnetic element.

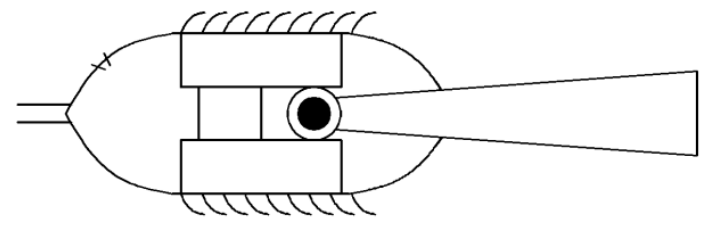

Figure 6. Design of Micro Robot

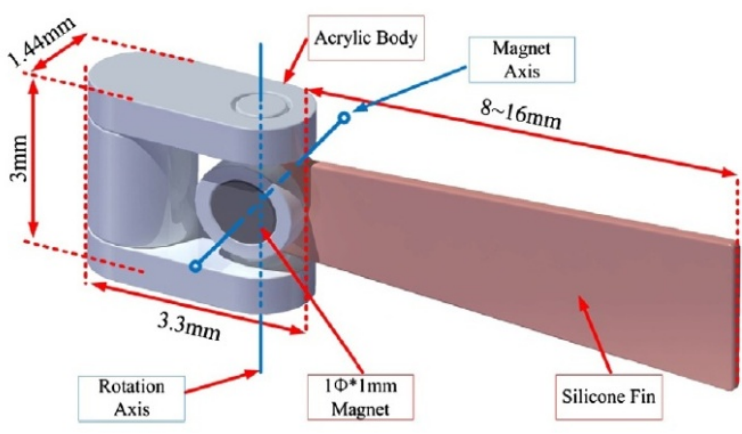

Figure 7 Isometric view of model 
As shown in Figure 6 the micro robot consists of an acrylic body, rotating axis, a cylinder type magnet and a silicone fin. The cylindrical magnet (diameter $1 \mathrm{~mm}$, height $2 \mathrm{~mm}$ ) with a high magnetic flux density is used. To convert the partial rotation of the magnet to the propulsion of the swimming micro robot, a silicone fin is attached at the rocking part installed on the permanent magnet. Firstly, the fin of the micro robot is aligned to the swimming direction. The magnetic axis is perpendicular to the swimming direction. The isometric view of the model is shown in Figure 7.
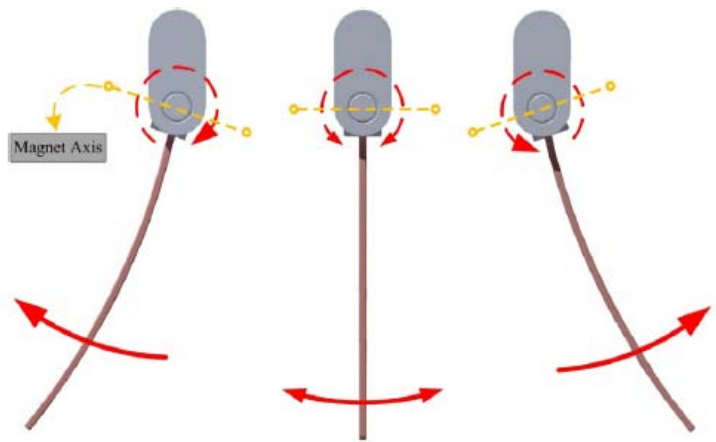

Figure 8. Schematic of Locomotion of robot

\section{RESULTS AND ANALYSIS OF THE MOVING DIRECTION AND PROPULSION}

The currents of the EMA coil system should be controlled to generate a magnetic field in the desired direction. The magnetic field generated by the two pairs of Helmholtz coil, which are positioned perpendicularly with each other, can be defined as the vector sum of the magnetic fields of the pair of Helmholtz coils. Along the desired direction, the uniform magnetic flux can be generated, and the permanent magnet can be aligned with the desired direction.

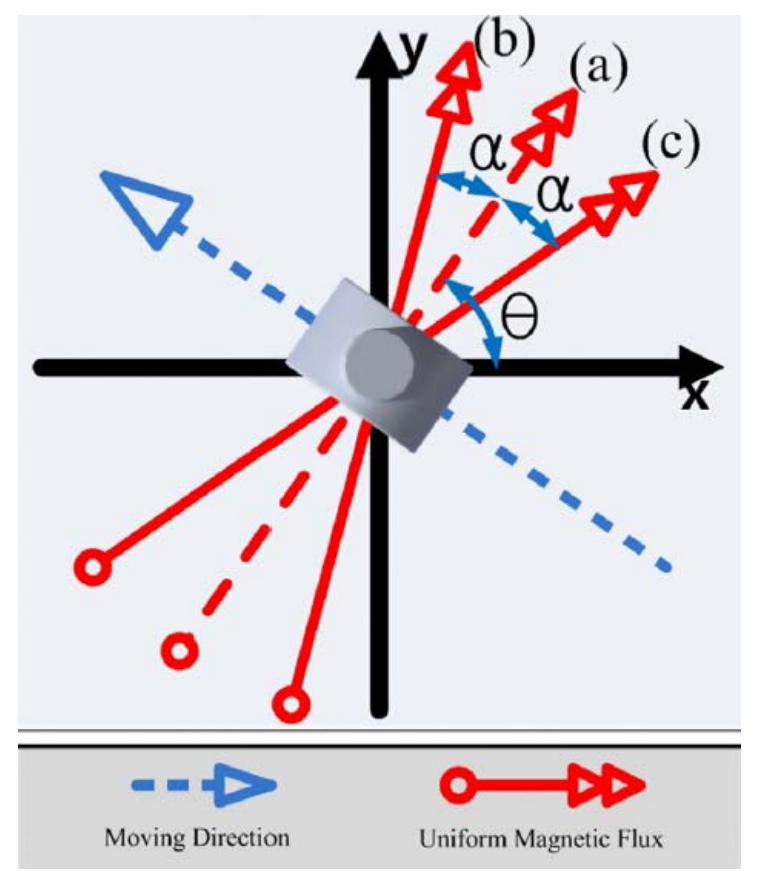

Figure 9. Analysis of the moving direction

In Figure 9 the blue arrow means the desired moving direction of the micro robot and the red arrow means the direction of the uniform magnetic field generated by the EMA coil system. Initially, the uniform magnetic field (a) is perpendicular with the desired moving direction and thus the permanent of the micro robot is aligned with the uniform magnetic field (a). Secondly, the uniform magnetic field is changed to direction (b), and the permanent magnet is aligned with direction (b). Similarly, the uniform magnetic field is changed to direction (c) and the permanent magnet is aligned with the direction (c). Therefore, when the 
permanent magnet of the micro robot is switched from (b) to (c), the fin attached with the magnet also swings with the magnet, and thus the micro robot shows swimming motion.

\subsection{Propulsion Mechanism Using Mathematical Model}

In detail, firstly, to generate the desired magnetic field of direction (a), the currents of two pairs of Helmholtz coils $\left(\mathrm{I}_{\mathrm{x}, \mathrm{a}}, \mathrm{I}_{\mathrm{y}, \mathrm{a}}\right)$ are set to

$$
\begin{aligned}
\mathrm{I}_{\mathrm{x}, \mathrm{a}} & =\mathrm{I}_{\max } \cos \theta \\
\mathrm{I}_{\mathrm{y}, \mathrm{a}} & =\mathrm{I}_{\max } \sin \theta,
\end{aligned}
$$

where $I_{\max }$ is the maximum input current. Secondly, when the magnetic field is aligned to direction (b), the currents of the two pairs of the Helmholtz coils are described as:

$$
\begin{aligned}
& \mathrm{I}_{\mathrm{x}, \mathrm{b}}=\mathrm{I}_{\max } \cos (\theta+\alpha) \\
& \mathrm{I}_{\mathrm{y}, \mathrm{b}}=\mathrm{I}_{\max } \sin (\theta+\alpha)
\end{aligned}
$$

Finally, in the aligned direction (c), the currents of the two pairs of the Helmholtz coils are described as:

$$
\begin{aligned}
I_{x, c} & =I_{\max } \cos (\theta-\alpha) \\
I_{y, c} & =I_{\max } \sin (\theta-\alpha)
\end{aligned}
$$

To change the direction of the magnetic field from (b) to (c) continuously, the term of $\alpha$ should be defined by the sinusoidal function $\alpha(\mathrm{t})=\alpha_{\max } \sin (\omega \mathrm{t})$, where $\alpha_{\max }$ is half switching angle of the fin and $\omega$ is the switching velocity of the swing fin. Therefore, finally, to generate a continuously switching magnetic field in the $\pm \alpha_{\max }$ direction the currents of the two pairs of the Helmholtz coils are defined as:

$$
\begin{aligned}
& I_{x}=I_{\text {max }} \cos [\theta+\alpha(t)] \\
& I_{y}=I_{\max } \sin [\theta+\alpha(t)]
\end{aligned}
$$

\subsection{Experimental Setup}

Figure 10 shows the schematics of the experimental setup in this study. The parts are shown in the figure. Two DC power supplies were adopted, and a relay circuit is installed to change the sign of the current. The final experimental setup is shown in Figure 11.

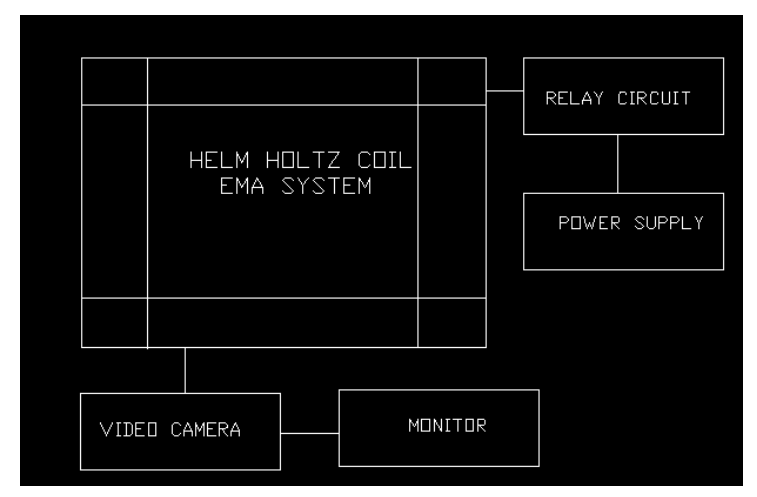

Figure 10. Block diagram of set up

\subsection{Experimental Results}

The performance of the swimming micro robot is evaluated by various experiments measuring the velocities of the micro robot according to variables such as the swing angle, the swing frequency, and the fin length. To verify the effect of the swing angle and the swing frequency on the performance of the micro robot, one of the variables is fixed as a constant value, and the other variable is changed as the micro robot swam, with the fin of the micro robot. 


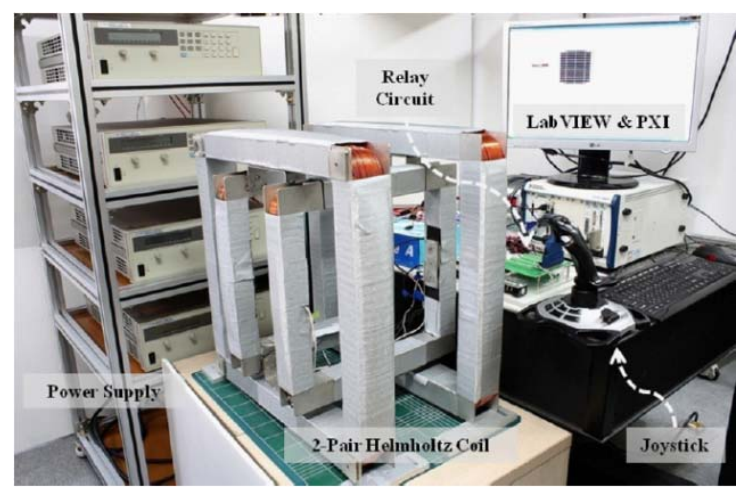

Figure 11. Experimental setup

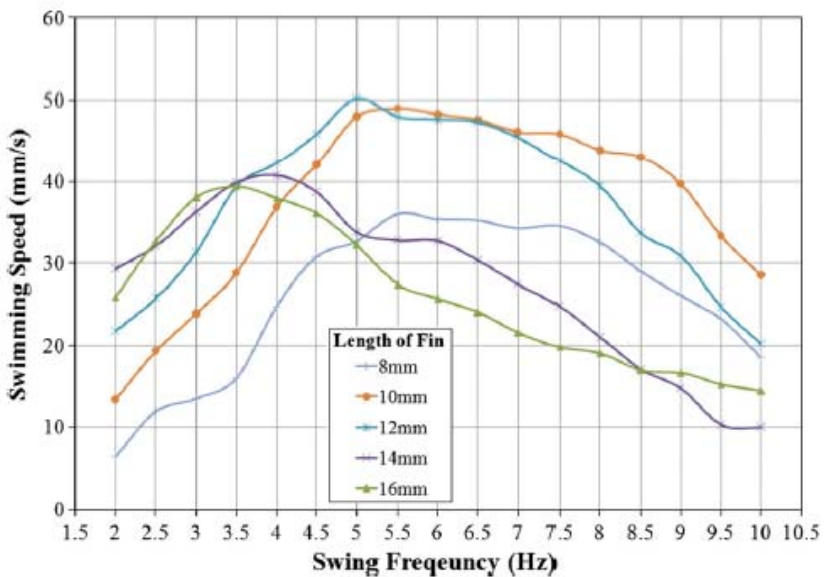

Figure 12. Swimming Speed VS Swing Frequency

Firstly, when the swimming velocity according to the swing frequency of the fin is measured, the swing angle is fixed as $80^{\circ}$. On the contrary, the swing frequency is set to $5 \mathrm{~Hz}$ to verify the effect of the swimming velocity according to the swing angle. For the measurement of the swimming velocity, the swimming distance is set to $100 \mathrm{~mm}$, and $\mathrm{I}_{\max }$ is set to $3.5 \mathrm{~A}$ in the experiments. In addition, the effect of the fin length on the swimming performance is verified by the changing of the fin length from $8 \mathrm{~mm}$ to $16 \mathrm{~mm}$.

Figure 12 shows the experimental graph on the swimming velocity according to the swing frequency. When the fin length is shorter than $12 \mathrm{~mm}$, the swimming micro robot shows fast and stable velocities in range of high frequencies (over $5 \mathrm{~Hz}$ ). On the contrary, the micro robot which has a longer fin length than $12 \mathrm{~mm}$ shows fast swimming velocities in the range of low frequencies (under $4 \mathrm{~Hz}$ ). Generally, the maximum swimming velocities appear in the frequency range between $4 \mathrm{~Hz}$ to $6 \mathrm{~Hz}$ and the significant decreases of the swimming velocity are shown after $8 \mathrm{~Hz}$. The micro robots with the short fin have fast and stable swimming motions in the range of high swing frequencies because their short fins decrease the effect of the momentum of the rotating parts including its fin under the same magnetic condition. However, when the fin is too short, the micro robot shows unstable swimming.

The swimming velocities according to the swing angle $\left(\alpha_{\max }\right)$ are shown in Figure 13 . The experimental graph shows that the swimming velocity increased with the swing angle of the magnetic flux. However, when the fin length of the micro robot is too short or too long, the performance of the micro robot deteriorated.

These experimental results show that the performance of the micro robot is seriously affected by the swing angle because the swing angle of the fin could not follow to the desired swing angle at the high swing frequencies. In addition, the fin length of the micro robot has a strong influence on the swimming motion and the velocity. Therefore, the micro robot with about $12 \mathrm{~mm}$ of the fin length shows the best velocity and stable swimming motion, as in Figs. From these results the optimized values are $\mathrm{I}_{\max }=3.5 \mathrm{~A}$, Swing frequency $=5$ $\mathrm{Hz}$, Swing angle $\left(\alpha_{\max }\right)=80^{\circ}$ and $12 \mathrm{~mm}$ of the fin length. 


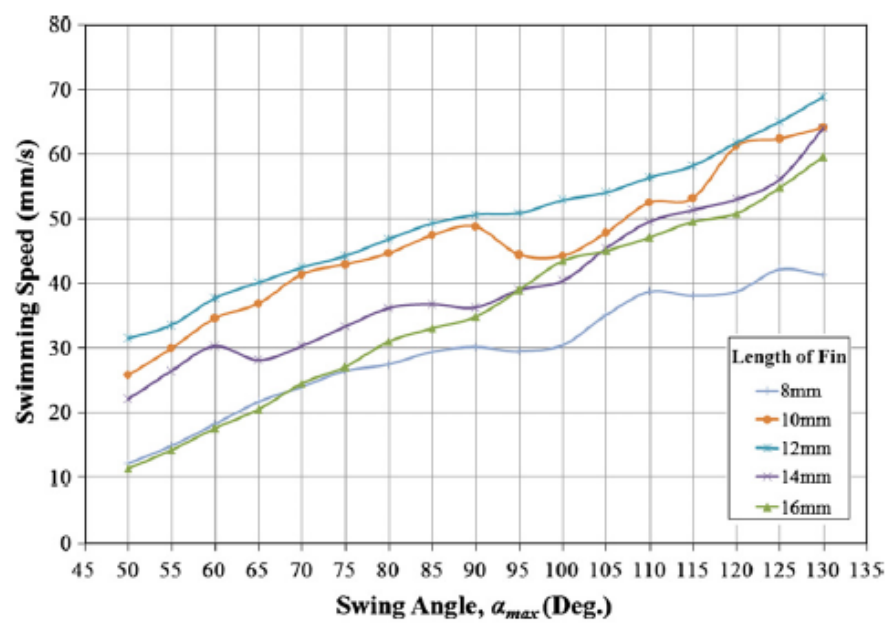

Figure 13. Swimming Speed VS Swing Angle

\section{CONCLUSION}

In this paper, firstly, the design, fabrication and control of the micro robots, current micro robot technology has been reviewed. Based on the review a new type of swimming micro robot for minimally invasive surgery has been proposed. Firstly, the design and structure of the micro robot and EMA coil system is presented. Secondly, the control mechanism for the micro robot has been derived. Then three variables are selected to modify the swimming performance of the micro robot in the control mechanism. Then, by various experiments the optimized values have been found. Developing this technology requires that we address issues such as localization and power. Effective collaboration between medical and robotics experts is needed.

\section{REFERENCES}

[1] Bradley J., Nelson ETH Zurich, "Microrobotics in Medicine" Institute of Robotics and Intelligent Systems Zurich, Switzerland, Review of Biomedical Engineering, 2008.

[2] Y S Zhou1*, Y X Quan1, K Yoshinaka2 and K Ikeuchi2 "A new medical microrobot for minimal invasive surgery" Proc. Instn Mech engrs Journal of Engineering in Medicine, vol.215, pp. 215-220, 2001.

[3] Kósa G, Jakab P, Nobuhiko Hata, Jólesz F, Zipi Neubach Z, Shoham M, "Flagellar Swimming for Medical Micro Robots: Theory, Experiments and Application" In: $2^{\text {nd }}$ IEEE international conference on biomedical robotics, $\mathrm{p}$. 258-263, 2007.

[4] Bradley J. Nelson,1 Ioannis K. Kaliakatsos, and Jake J. Abbott2 "Micro robots for Minimally Invasive Medicine". The annual review of Biomedical engineering, pp.55-85, 2010.

[5] ViRob- "An Autonomous Crawing Micro-robot". The Technion, Technology institute, Israel, May, 2008.

[6] Taheri A, Meysam, Moosavy S, "A Numerical Strategy to Design Maneuverable Micro-Biomedical Swimming Robots Based on Bio mimetic Flagellar Propulsion", World Academy of science and technology, Vol .54, pp.500504, 2009.

[7] Dumsong E, Afzulpurkar N, Tuantranont A and Punyasai C, "Design and Simulation of Wireless, Walking ScratchDrive Micro-Robot", 10 Intl. Conf. On Control, Automation, Robotics and Vision, p.588-592, 17-20 Dec, 2008.

[8] B. Behkam, M. Sitti, "Design Methodology for biomemitic propulsion of miniature Swimming Robots," J. Dynamic Systems Measurement and Control, Vol. 128, pp.136-43, 2006.

[9] J. B. Moidel, J.D. Ozer, O. Kuter-Arnebeck "Water Actuation of Micro Robotics", Journal of Micro/ Nano Robotics, pp.43-48, 2006.

[10] Wei Zhang_Shu-Xiang Guo “A New Type of Hybrid Fish-like Micro-robot”, Intl. Journal of Automation and computing, Vol.4, pp.358-365, 2006.

[11] S. Guo, Y. Hasegaw, T. Fukuda, and K. Asaka, "Fish -Like underwater microrobot with multi DOF," Proc. of 200 International Symposium on Micromechatronics and uman Science, pp. 63-68, 2001.

[12] Christophe Perrard, Nicolas Andreff "Control of a team of micro-robots for non-invasive medical applications", Proc. $6^{\text {th }}$ National conf. On control Architecture of robots, CAR'11, July 2011.

[13] Huaming Li and Jindong Tan Mingjun Zhang "Dynamics Modeling and Analysis of a Swimming Microrobot for Controlled Drug Delivery". Proc. IEEE International conference on Robotics and Automation, pp.1768-1773, 2006.

[14] Guo S, Pan Q. Mechanism and control of a novel type microrobot for biomedical application. In: IEEE international conference on robotics and automation; p. 187-92, 2007. 
[15] Savvas G. Loizou, Kostas J. Kyriakopoulos, "Motion Planning of Piezoelectrically Driven Micro-Robots via Navigation Functions" Springer, 1997.

[16] Dario, P., Guglielmelli, E., Allotta, B. and Carrozza, M. C. "Robotics for medical applications". IEEE Robotics AutomnMag., Vol.3, pp.44-56, 1996.

[17] P. Dario, M.C. Carrozza, L. Lencioni, B. Magnani, C. Filippeschi, M.G. Trivella, A. Pietrabissa, "A Microrobot System for Lower Gastrointestinal Inspection and Intervention”. Sensors and Microsystems, pp.14-21, Singapore, 1996.

[18] Peter J. Berkelman, Louis L. Whitcomb, Russell H. Taylor, and Patrick Jensen "A Miniature Microsurgical Instrument Tip Force Sensor for Enhanced Force Feedback During Robot-Assisted Manipulation". Proc. IEEE Transactions on Robotics and Automation, Vol.19, no. 5, pp.917-922, Oct, 2003.

[19] Yuan Zheng, George Bekey, Arthur Sanderson "Robotics for biological and medical applications", Intl. Journal of Emerging Medical Technologies, Jan27, 2006.

[20] Hülsen H, Trüper T, Fatikow S, "Control System for the automatic Handling of biological Cells with mobile Micro robots”, Proc. American Control Conference, pp.3986-3991, July 2, 2004.

[21] Tomie M, Takiguchi A, Honda T, Yamasaki J. "Turning performance of fish-type micro robot driven by external magnetic field", IEEE transactions on Magnetics 2005; 4015-7.

[22] ArthurW. Mahoney John C. Sarrazinb, Eberhard Bamberg b and Jake J. Abbott "Velocity Control with Gravity Compensation for Magnetic Helical Microswimmers", In: Advanced Robotics, no.25, pp.1007-1028, 2011.

[23] Joo Han Kim*, Se Hyun Rhyu, In Soung Jung, Jung Moo Seo "An investigation on development of Precision actuator for small robot", Proc. $9^{\text {th }}$ WSEAS Intl.Conf. on Robotics, Control and Manufacturing technology, pp. 62$66,2008$.

[24] Joseph JV, Arya M, Patel HRH, "Robotic surgery: the coming of a new era in surgical innovation”, Expert Rev. Anticancer Ther. 5(1):7-9, 2005.

[25] G. T. A. Kovacs, "Micromachined Transducers Sourcebook". WCB/McGaw-Hill, ISBN, 0-07-290722-3, 1998.

[26] K.B. Yesin, B.J. Nelson, "Robust CAD Model Based Visual Tracking for 3D Microassembly Using Image Space Potentials". Proc. IEEE International conference on Robotics and automation, pp.1868-1873, 2004.

[27] Nagy Z, Ergeneman O, Abbott JJ, Hutter M, Hirt AM, Nelson BJ. "Modeling assembled-MEMS microrobots for wireless magnetic control”, Proc. IEEE Int. Conf. Robot. Autom., Pasadena, Calif., May 19-23, pp. 874-79, 2008.

[28] Vilkomerson D, Lyons D. 1997. "A system for ultrasonic beacon-guidance of catheters and other minimally invasive (in the original title, they write "minimally-invasive") medical devices", IEEE Trans. Ultrason. Ferroelectr. Freq. Control 44(2):496-504.

[29] B. Behkam and M. Sitti. "Modeling and Testing of a Biomimetic Flagellar Propulsion Method for Microscale Biomedical Swimming Robots", ASME J. Dyn. Syst. Meas. Control, 2006.

[30] Guo S, Pan Q, Li D. "Mechanism and control of a spiral type of microrobot in pipe. In: IEEE conference on robotics and biomimetics", p.43-48, 2008.

[31] Frank Tendick, S. Shankar Sastry, Ronald S. Fearing and Michael Cohn, "Applications of Micromechatronics in Minimally Invasive Surgery” Proc. IEEE/ASME transactions on mechatronics, vol. 3, no. 1, p. 34-42, March 1998.

[32] Guo S, Sawamoto J, Pan Q. "A novel type of microrobot for biomedical application". In: International robots and systems, p. 1047-1052, 2005.

[33] Abbott JJ, Nagy Z, Beyeler F, Nelson BJ. "Robotics in the small-part I: micro robotics". IEEE Rob Autom Mag 2007, 14: p. 92-103.

[34] Honda T, Arai KI, Ishiyama K. "Micro swimming mechanisms propelled by external magnetic fields", IEEE Trans. Magn. 32(5):5085-87, 1996.

[35] Dogangil G, Ergeneman O, Abbott JJ, Pan'e S, Hall H, 2008. "Toward targeted retinal drug delivery with wireless magnetic microrobots", Proc. IEEE/RSJ Int. Conf. Intell. Robots Syst., Nice, Fr., Sept. 22-26, pp. 1921-26, 2008.

[36] T. Fukuda, A. Kawamoto, F. Arai, and H. Matsuura, "Mechanism and swimming experiment of micro mobile robot in water," Proc. of IEEE Int'l Workshop on Micro Electro Mechanical Systems (MEMS'94), pp.273-278.

[37] J. Edd, S. Payen, B. Rubinsky; M.L. Stoller and M. Sitti, "Biomimetic propulsion for a swimming surgical microrobot," IEEE/RSJ Intelligent Robotics and Systems Conference, vol. 3, pp. 2583 - 2588, October 2003.

[38] S. Guo, Y., Okuda and, K. Asaka, "Hybrid type of underwater micro biped robot with walking and swimming motions" In Proceedings of IEEE Intl. Conf. On Mechatronics and Automation, Ontario, Canada, pp.81-86, 2005.

[39] K.B. Yesin, K. Vollmers, and B.J. Nelson, "Modeling and control of untethered biomicrorobots in a fluidic environment using electromagnetic fields," Int'l J.Robotics research, vol.25, no.5-6, pp.527-536, 2006.

[40] Haga Y, Esashi M. "Biomedical microsystems for minimally invasive diagnosis and treatment", Proc. IEEE 92(1):98-114, 2004.

[41] Y. Zhang, Q. Wang, P. Zhang, X. Wang, and T. Mei, "Dynamic analysis and experiment of a 3mm swimming microrobot," Proc. of the 2004 IEEE/RSJ International Conference on Intelligent Robots and Systems, pp. 17461750,2004

[42] Byun D, JonghoChoi, Kyoungae Cha "Swimming micro robot actuated by two pairs of Helmholtz coils system", Journal of Mechatronics, pp. 357-364, 2011.

[43] ZHOU Yinsheng1, HE Huinong1, GU Daqiang1, AN Qi2 \& QUAN Yongxin1 "Noninvasive method to drive medical micro-robots" Chin. Sci. Bull., vol.45, pp.617-620, 2000.

[44] B. L. Davies, "A discussion of safety issues for medical robots," in Computer-Integrated Surgery: Technology and Clinical Applications, R. H. Taylor, S. Lavallee, G. C. Burdea, and R. Mosges, Eds. Cambridge, MA: MIT Press, 1996, pp. 287-298. 
[45] Bahareh Behkam, Metin Sitti “ Coli Inspired Propulsion For Swimming Micro robots”, Carnegie Mellon University, Proc. International Mechanical Engg. Conference and R\&D Exposition, Nov 13-19, 2004.

[46] G. Kósa, M. Shoham and M. Zaaroor., "Propulsion Method for Swimming Micro Robots", IEEE Transaction on Robotics, vol. 23, pp. 137-150, Jan. 2007.

[47] Michael Sfakiotakis, David m. Lane, and j. Bruce c. Davies, "Review of fish swimming modes For aquatic locomotion", IEEE journal of oceanic Engineering, vol. 24, no. 2, April 1999.

[48] H. K. Cheng and L. E. Murillo, "Lunate-tail swimming propulsion as a problem of curved lifting line in unsteady flow. Part 1. Symptotic theory," J. Fluid Mech., vol. 143, pp. 327-350, 1984.

[49] T. Nakaoka and Y. Toda, "Laminar flow computation of fish-like motion wing," in Proc. 4th Int. Offshore and Polar Eng. Conf., Osaka, Japan, Apr. 1994, pp. 530-538.

[50] Popovic M, Popovic BD, Popovic Z. 2006. Electromagnetic induction. In Fundamentals of Engineering Electromagnetics, ed. R Bansal. Boca Raton, FL: Taylor \& Francis

[51] Siauve N, Scorretti R, Burais N, Nicolas L, Nicolas A. 2003. Electromagnetic fields and human body: a new challenge for the electromagnetic field computation. Int. J. Comput. Math. Electr. Electron. Eng. 22(3):457-69

[52] S. Guo, N. Kato, T. Fukuda, and K. Oguro, "A fish-microrobot using ICPF actuator," in Proc. 1998 5th Int. Workshop on Advanced Motion Control, Coimbra, Portugal, June 1998, pp. 592-597.

[53] Behkam B, Sitti M. 2006. Design methodology for biomimetic propulsion of miniature swimming robots. ASME J. Dyn. Syst. Meas. Control 128(1):36-43

[54] Yesin KB, Vollmers K,Nelson BJ. 2006. Modeling and control of untethered biomicrorobots in a fluidic environment using electromagnetic fields. Int. J. Robot. Res. 25(5-6):527-36

[55] G. V. Lauder and B. C. Jayne, "Pectoral fin locomotion in fishes-Testing drag-based models using 3-dimensional kinematics," Amer. Zool., vol. 36, pp. 567-581, 1996.

[56] F. Cepolina. Development of micro tools for surgical applications. Ph.D. Thesis, Universita' Degli Studi De Genova/ Uiversite Piere Et marie Currie Paris, 2005.

[57] N. Zemiti and al "Mechatronic Design of a New Robot for Force Control in Minimally Invasive Surgery", IEEE/ASME Transactions on Mechatronics, vol 12(2), april 2007,pp 143-153.

[58] Tobias Ortmaier and Gerd Hirzinger. Cartesian Control Issues for Minimally Invasive Robot Surgery. In Proc. of the IEEE/RSJ International Conference on Intelligent Robots and Systems IROS 2000, Takamatsu, Japan, October 2000 .

\section{BIOGRAPHY OF AUTHOR}

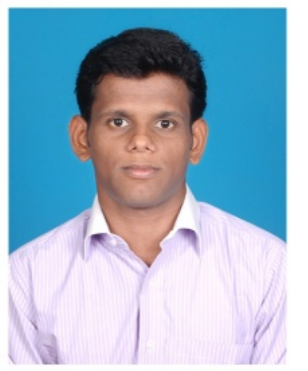

Deiva Ganesh.A got his undergraduate degree in Mechanical Engineering from Mepco Schlenk Engineering College, Anna University Chennai in 2008. Later he worked as a Lecturer in an Engineering College in Coimbatore, TamilNadu for two years. Then he pursued his Master's Degree in Engineering Design, Mechanical Engineering from Sona College of Technology, Anna University Coimbatore in 2012. His research interests are Engineering Design and Robotics. Currently he is working as Assistant Professor in the Department of Mechanical Engineering, Velammal Engineering College, Chennai, Tamil Nadu, India. 